\title{
Effect of pollution severity and dry band location on the flashover characteristics of silicone rubber surfaces
}

\author{
Arshad $^{1}$ - A. Nekahi ${ }^{1}$ - S. G. McMeekin ${ }^{1}$ - M. Farzaneh ${ }^{2}$
}

Received: 7 March 2016 / Accepted: 2 November 2016 / Published online: 10 November 2016

(C) The Author(s) 2016. This article is published with open access at Springerlink.com

\begin{abstract}
This paper investigates the flashover and ageing behavior of silicone rubber insulators under various contamination and dry band conditions. Effect of pollution severity, leakage distance and dry band location on the flashover characteristics of silicone rubber insulator was investigated. Flashover voltage and electric field distribution were measured under different pollution severity levels and dry band conditions. Effect of leakage distance on flashover voltage was also studied. Tests were carried out in a climate chamber and specific values of humidity and ambient temperature were used. A commercially available simulation package, COMSOL Multiphysics, was used to validate the experimental results. Electric field and potential along a silicone rubber sample were studied under different pollution severity and dry band conditions. Fourier transform infrared spectroscopy was used to analyze the chemical changes on the insulator surface and investigate the hydrophobicity recovery property of silicone rubber after flashover tests. The results of this study will further our knowledge regarding the flashover of silicone rubber outdoor insulators under contaminated and dry band conditions and could be used to improve the existing flashover models.
\end{abstract}

Arshad

arshad@gcu.ac.uk

M. Farzaneh

http://www.cigele.ca

1 School of Engineering and Built Environment, Glasgow Caledonian University, Glasgow G4 0BA, UK

2 Canada Research Chair on Atmospheric Icing Engineering of Power Networks (INGIVRE), Université du Québec à Chicoutimi, Chicoutimi, QC, Canada
Keywords Silicone rubber · FTIR · Pollution · Flashover · Dry bands · Electric field

\section{Introduction}

During the past few decades, silicone rubber insulators have been used extensively as high-voltage outdoor insulators. Due to their hydrophobic nature, their performance is considered to be superior to that of porcelain and glass insulators. The high contamination-withstanding characteristics of silicone rubber are due to the diffusion of low-molecular-weight (LMW) molecules on the surface [1]. Silicone rubber also offers the advantage of light weight and easy handling, but due to their organic nature, ageing is unavoidable. Ageing of silicone rubber insulators due to ultra-violet radiation has been studied by many researchers [2-5]. Their surface chemistry and hydrophobicity loss due to various environmental factors have been studied in [6-9].

Ageing of polymeric insulators is influenced by many factors: wet pollution, Joule heating due to current flow, rain, ultraviolet radiation and dry band arcing [10]. It has been found that the flashover process for polymeric insulators is different than that of porcelain and glass insulators [11]. During mist, light rain or fog, water droplets also form on polluted insulator surfaces. The soluble contaminants present in the pollution layer dissolve in water droplets and increase their conductivity. The high electric field at the junction of water droplets, insulator surface and air causes the water droplets to become elongated in the direction of the electric field. This elongation of water droplets leads to the formation of a conductive channel with neighboring droplets and water filaments along the insulator surface. The discharge initiated between these filaments often leads to a loss of hydrophobicity and the formation of a conduc- 
tive water channel on the insulator surface. This conductive water film leads to an increase in leakage current which causes the formation of dry bands along the insulator surface [12]. It was reported in [13] that the threshold value for dry and wet corona initiation is 2.28 and $0.45 \mathrm{kV} / \mathrm{mm}$, respectively.

Pioneering work on pollution flashover was done by Obenaus [14]. Obenaus did not consider arc re-ignition conditions and assumed that flashover only occurs if the insulator surface is completely bridged by the arc without extinguishing. Most of the current flashover models for porcelain and glass insulators are based on the Obenaus model [15-18]. Usually, IEC 60507 [19] is used for artificial pollution test of outdoor insulators. This method is applicable for porcelain and glass insulators but cannot be used for polymeric insulator without certain modifications. For polymeric insulators, a modified solid layer method based on IEC 60507 was proposed in $[20,21]$. This method requires preconditioning with dry powder and the addition of a wetting agent in the pollution suspension.

Pollution deposition on outdoor insulators is not uniform and is influenced by many factors, e.g., wind direction and speed, insulator geometry and orientation etc. The nonuniform pollution deposition along an outdoor insulator surface leads to non-uniform current density and power dissipation. The flow of leakage current heat up the insulator surface, and due to the non-uniform current density, some parts of the insulator dry out faster than the others. This phenomenon leads to the formation of dry bands. The flashover characteristics of outdoor insulators are influenced by the formation of dry bands and have been investigated in the literature $[22,23]$. Based on the results presented in reference [22], the dry band width is dependent on various parameters, e.g., pollution distribution, current density, applied voltage, and fog rate. The average dry band width reported in [22] is $8-10 \%$ of the creepage distance. Electric field enhancement due to dry band formation has been investigated in [24]. The thermal effect of dry band discharges on the surface ageing of polymeric insulators has been studied in [25].

In this study, the modified solid layer method was used to pollute samples artificially in laboratory. Four pollution suspensions were prepared with volume conductivities of $1.4,4.0,8.0$ and $16.0 \mathrm{~S} / \mathrm{m}$ corresponding to light, medium, heavy and very heavy pollution severity levels, respectively. Numerical simulations were performed with COMSOL Multiphysics to calculate the electric field and potential distribution along the insulator surface at different pollution severity levels and dry band locations. To simulate light, medium, heavy and very heavy pollution, a 2 -mm-thick water layer was applied on the insulator surface and the conductivity of the layer was varied to model different polluted environments. To analyze the chemical changes on the insu- lator surface after flashover tests, FTIR spectroscopy was used.

The main objectives of this paper are outlined below:

- Investigate the effect of pollution severity on critical flashover voltage, electric field and potential distribution.

- Investigate the effect of dry band location and leakage distance on critical flashover voltage and electric field distribution.

- Study the hydrophobicity recovery property of silicone rubber insulators after performing flashover tests.

\section{Experimental setup}

Simplified test setup diagram is shown in Fig. 1. A 10-cmlong, 4-cm-wide and 6-mm-thick plate sample of silicone rubber was chosen for the experiment. Voltage was applied through a $100 \mathrm{kVA}, 0-100 \mathrm{kV}$, and $50 \mathrm{~Hz}$ transformer. The overvoltage was configured at $50 \mathrm{kV}$ and the maximum test current was set at $1 \mathrm{~A}$. The voltage was applied using a copper hemispherical electrode of $1.2 \mathrm{~cm}$ diameter at the high-voltage end and a rectangular copper plate at the ground end. Both the electrodes were attached to the silicone rubber surface using copper screws, and the sample was placed on a Plexiglas surface horizontally. This electrode configuration was chosen to minimise any unwanted corona activity due to sharp edges near the electrode ends and obtain a stable arc between the two electrodes which will enable us to obtain the time-resolved spectra of flashover arc using optical emission spectroscopy. To measure the voltage across the insulator sample, a capacitive divider was used. Leakage current was measured using a current transformer (CT). A National Instruments' (NI) data acquisition card (DAQ) was

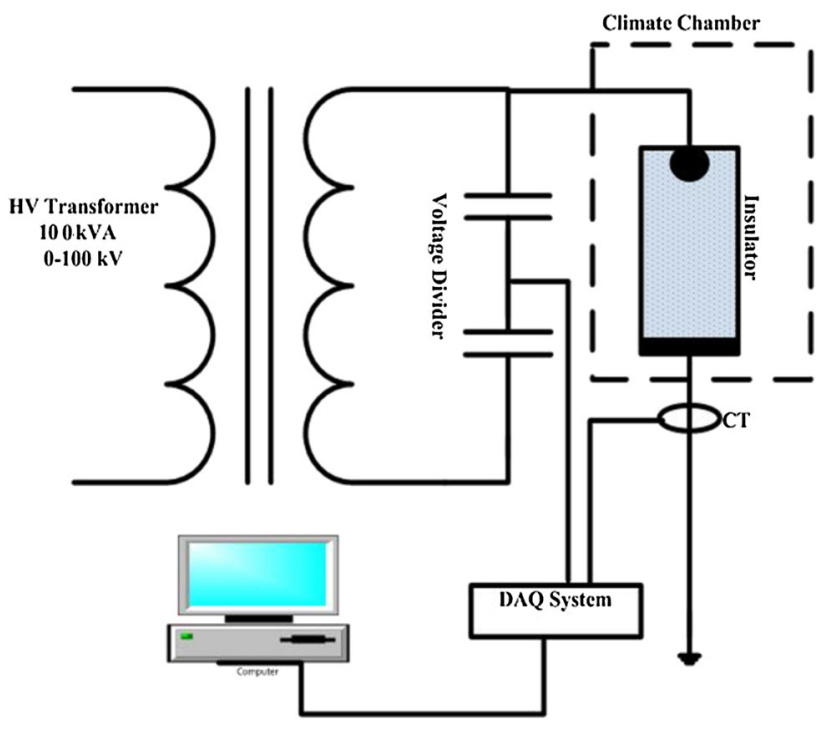

Fig. 1 Circuit diagram of test setup 
used to record the current and voltage signal and store data for further processing. A LabVIEW program was designed to measure current and voltage signals during the tests. Voltage and current data were stored in sinusoidal as well as root mean square (RMS) form. An FTIR spectrometer was used to analyse the surface changes after flashover tests. Hydrophobicity recovery property of silicone rubber was investigated by taking FTIR measurements after certain intervals.

\section{Test method}

All tests were carried out in a state-of-the-art climate chamber having humidity, temperature and fog rate control. The samples were placed in a climate chamber having dimensions of $2.5 \mathrm{~m} \times 2.5 \mathrm{~m} \times 2 \mathrm{~m}$, and clean fog was generated at a rate of $4 \mathrm{l} / \mathrm{h}$. The average relative humidity and temperature in Scotland are $80 \%$ and $10{ }^{\circ} \mathrm{C}$, respectively, during the summer. In this paper, all tests were carried out at $80 \%$ relative humidity and ambient temperatures of $10{ }^{\circ} \mathrm{C}$ to replicate the field conditions in Scotland. Up to 20 min were allowed for wetting the insulator sample before energising. For dry band experiments, the dry band location was masked using an acrylic glass strip and removed after the wetting was completed. In this experiment, voltage was applied in steps of 5\% of the predicted flashover voltage starting from zero until the flashover occurs. Each step was maintained for 2 min, and if no partial arcs appeared, the voltage was increased. In the case of partial arc, the voltage was maintained at the same value until the arc disappeared or leads to flashover. Each test was repeated at least 10 times, and flashover voltages were recorded. The flashover voltage from each test was expressed as. The minimum flashover voltage and standard deviation were obtained using Eqs. (3.1)-(3.3). To improve the accuracy of the obtained results, several effective minimum flashover voltages are obtained and average values are presented.

$$
\begin{aligned}
& V_{\min }=\operatorname{Min}\left(V_{1}, V_{2}, \ldots \ldots, V_{n}\right) \\
& V_{\mathrm{av}}=\frac{\sum_{i=1}^{N} V_{\min }}{N} \\
& \sigma(\%)=\sqrt{\frac{\sum_{i=1}^{N}\left(V_{\min }(i)-V_{\mathrm{av}}\right)^{2}}{N}} \times \frac{100}{V_{\mathrm{av}}}
\end{aligned}
$$

\section{Test samples}

The hydrophobic nature of polymeric insulators makes it difficult to apply a uniform pollution layer on their surfaces. Unlike porcelain and glass insulators, there is no international standard available, and most of the previous work is

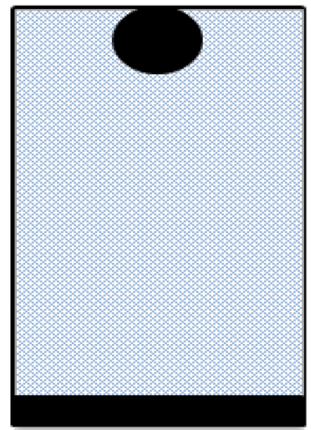

(a)

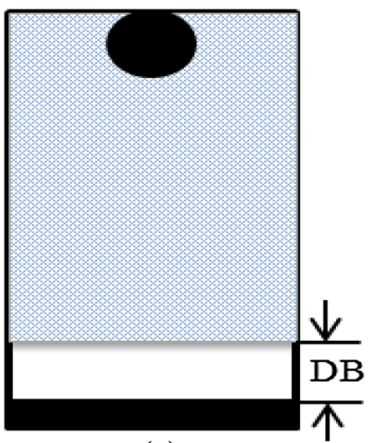

(c)

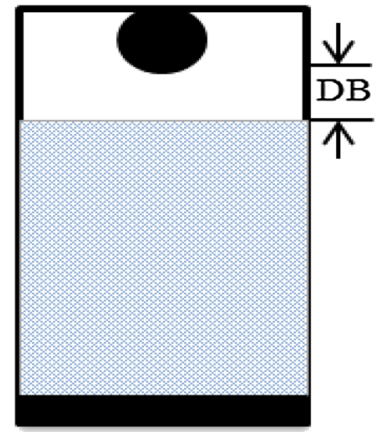

(b)

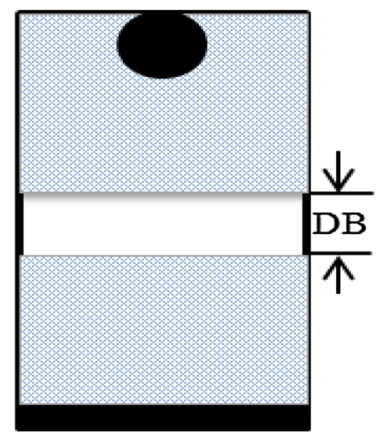

(d)
Fig. 2 Sample configurations a uniform pollution, b dry band at HV end, $\mathbf{c}$ dry band at GND end, $\mathbf{d}$ dry band in middle

based on water droplets instead of a uniform pollution layer. A modified artificial pollution method for polymeric insulators proposed in $[20,21]$ was applied here. This method is based on the solid layer procedure of IEC 60507 with Triton X-100 as an additional constituent in the pollution suspension. Triton X-100 is a wetting agent and helps in the uniform wetting of insulator surface. Another modification is the preconditioning of insulator surface with dry powder before applying the pollution suspension. Preconditioning was performed using dry kaolin on the insulator surface using a brush until the surface became hydrophilic. After preconditioning, the samples were checked for wettability class to ensure that the surface was hydrophilic. Samples were kept in open air for one hour before the application of pollution suspension. Pollution suspensions were prepared by adding $40 \mathrm{~g}$ of kaolin, $1 \mathrm{~g}$ of Triton X-100 and appropriate amount of sodium chloride in 11 of tap water. Pollution suspensions with volume conductivity of $1.4,4.0,8.0$, and $16 \mathrm{~S} / \mathrm{m}$ corresponding to light, medium, heavy and very heavy pollution levels, respectively, were prepared and applied on the insulator surfaces. Dry bands were inserted at the high-voltage end, ground end and middle parts of the sample as shown in Fig. 2. Length of each dry band was chosen to be $1 \mathrm{~cm}$ in each case. Polluted samples were kept in open air for $24 \mathrm{~h}$ before performing the flashover tests. Volume conductivities and ESDD for light, medium, heavy and very heavy pollution are given in Table 1. 
Table 1 Pollution severity

\begin{tabular}{lll}
\hline Pollution level & $\begin{array}{l}\text { ESDD } \\
\left(\mathrm{mg} / \mathrm{cm}^{2}\right)\end{array}$ & $\begin{array}{l}\text { Volume conductivity } \\
(\mathrm{S} / \mathrm{m})\end{array}$ \\
\hline Light & 0.035 & 1.4 \\
Medium & 0.1 & 4 \\
Heavy & 0.2 & 8 \\
Very heavy & 0.4 & 16 \\
\hline
\end{tabular}

Table 2 Flashover voltages and electric field

\begin{tabular}{lll}
\hline Pollution severity & $\begin{array}{l}\text { Flashover } \\
\text { voltage }(\mathrm{kV})\end{array}$ & $\begin{array}{l}\text { Standard } \\
\text { deviation }(\%)\end{array}$ \\
\hline Clean and dry & 27.5 & 1.8 \\
Clean and wet & 21.0 & 2.3 \\
Light & 11.28 & 3.7 \\
Medium & 10.2 & 2.5 \\
Heavy & 8.7 & 3.2 \\
Very heavy & 6.8 & 2.6 \\
\hline
\end{tabular}

\section{Experimental results}

\subsection{Effect of pollution severity}

Initially, the tests were performed on clean samples with dry and wet surfaces. Tap water was used to wet the insulator surface. Due to the hydrophobic nature of silicone rubber insulators, water droplets formed on the insulator surface. It was noted down that even without any pollution, the flashover resistance of polymeric insulators decreases as the wetting rate and number of water droplets increase. Performance of silicone rubber insulators under polluted conditions was analyzed by carrying out experiments on light, medium, heavy and very heavily polluted samples. Distance between electrodes was chosen to be $8 \mathrm{~cm}$ in this section. Several readings were taken and presented in Table 2 . The results obtained from experiments show that the standard deviation is between $1.8-3.7 \%$ and is independent of the pollution severity. Although several readings were taken during the experiment, this deviation could possibly be due to the variation in applying the artificial pollution layer, the surface pre-processing, or the wetting rate. The flashover voltage values obtained show that pollution severity has strong influence on the performance of silicone rubber insulators. Figure 3 shows the relationship between pollution severity, leakage current and flashover voltage.

\subsection{Effect of leakage distance}

The dielectric strength of insulator is influenced by the leakage distance. With increase in leakage distance, the insulation resistance increases and so does the flashover strength. In this

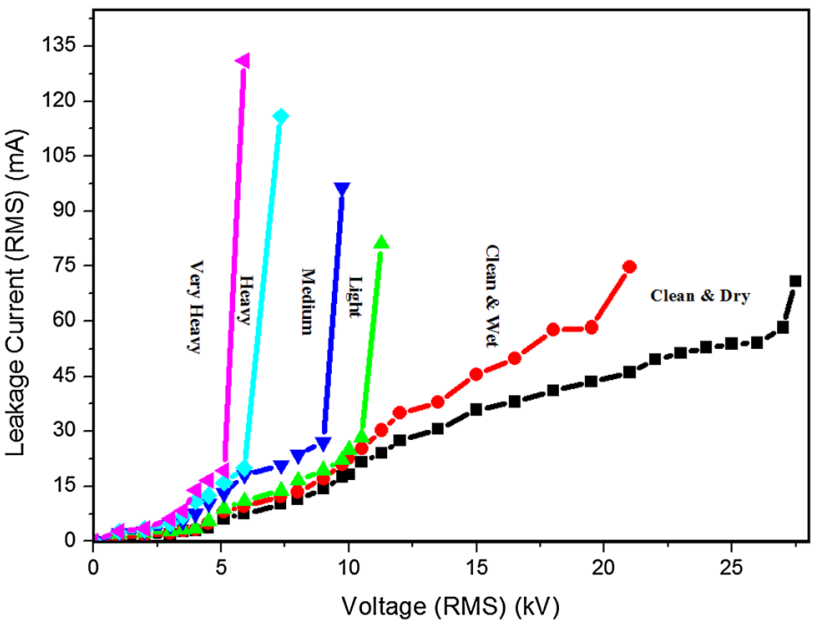

Fig. 3 Variation of leakage current with pollution severity

Table 3 Flashover voltages and electric field at different leakage distance

\begin{tabular}{lll}
\hline $\begin{array}{l}\text { Leakage } \\
\text { distance }(\mathrm{cm})\end{array}$ & $\begin{array}{l}\text { Flashover } \\
\text { voltage }(\mathrm{kV})\end{array}$ & $\begin{array}{l}\text { Standard } \\
\text { deviation }(\%)\end{array}$ \\
\hline 1 & 1.3 & 3.2 \\
2 & 2.7 & 4.0 \\
3 & 4.0 & 2.9 \\
4 & 5.35 & 3.1 \\
5 & 6.45 & 3.0 \\
6 & 8.0 & 4.5 \\
7 & 9.17 & 2.8 \\
8 & 10.25 & 2.7 \\
\hline
\end{tabular}

section, experimental results on flashover voltage and electric field are presented for a medium polluted silicone rubber sample. Distance between the electrodes is varied from 1 to $8 \mathrm{~cm}$ in steps of $1 \mathrm{~cm}$ and results are presented in Table 3 . An increase in flashover voltage with increase in leakage distance was observed. This variation may be due to the manual setting of the electrode distance, wetting rate and pollution application. The standard variations of flashover voltage are between 2.7 and $4.5 \%$. Several readings were taken to improve the accuracy of the results, and the higher percentage of deviation at $3-\mathrm{cm}$ and $6-\mathrm{cm}$ electrode distances could be due to manual pollution applications and pre-conditioning of the insulator surface (Fig. 4).

\subsection{Effect of dry band location}

The formation of dry bands on outdoor insulators is a natural phenomenon and cannot be eliminated completely. Pollution deposition on outdoor insulators depends on various factors and is non-uniform. Non-uniform pollution deposition on the insulator surface leads to non-uniform current den- 


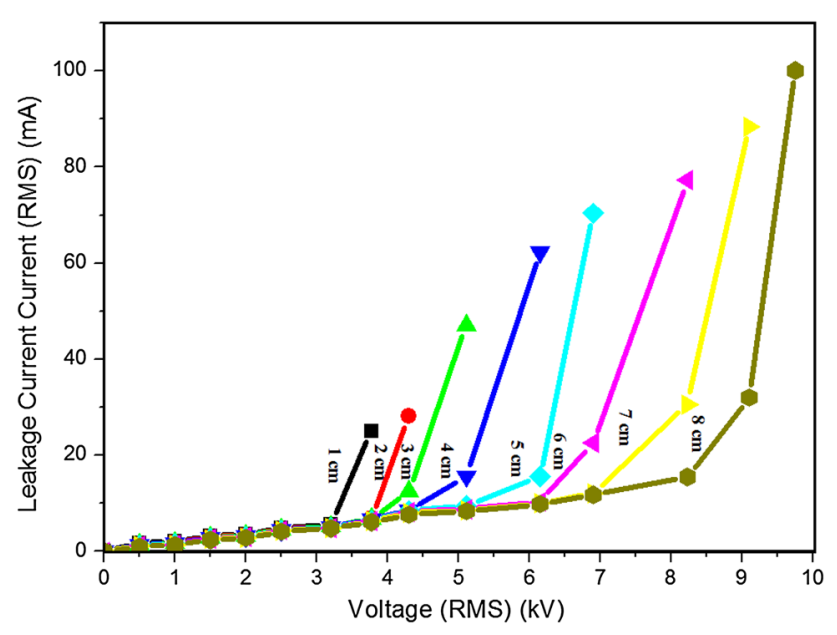

Fig. 4 Variation of leakage current with leakage distance

Table 4 Flashover voltages and electric field at different dry band location

\begin{tabular}{llll}
\hline $\begin{array}{l}\text { Pollution } \\
\text { severity }\end{array}$ & $\begin{array}{l}\text { Dry band } \\
\text { location }\end{array}$ & $\begin{array}{l}\text { Flashover } \\
\text { voltage }(\mathrm{kV})\end{array}$ & $\begin{array}{l}\text { Standard } \\
\text { deviation }(\%)\end{array}$ \\
\hline Light & HV & 12.3 & 2.2 \\
& Middle & 12.8 & 2.4 \\
& GND & 12.4 & 2.2 \\
Medium & HV & 11.25 & 2.5 \\
& Middle & 11.8 & 2.2 \\
& GND & 11.4 & 3.2 \\
Heavy & HV & 9.72 & 2.8 \\
& Middle & 10.3 & 2.6 \\
& GND & 9.85 & 3.1 \\
Very heavy & HV & 7.8 & 2.5 \\
& Middle & 8.35 & 2.3 \\
& GND & 7.9 & 2.8 \\
\hline
\end{tabular}

sity and formation of dry bands. Partial arcs and flashover models for polluted insulator having dry bands have been developed mathematically and in laboratory $[22,26]$. Experimental investigations were carried out in this paper to study the effect of dry band location on flashover voltage of silicone rubber insulators. Three types of configuration were used for the tests as shown in Fig. 2. A 1-cm dry band was inserted at the energised end, ground end and middle part of the insulator. The results obtained in this section were compared to those of section 4.1 and it was found that dry band formation increases flashover voltage. Furthermore, it is evident from Table 4 that flashover voltage is high in the case of a dry band in the middle region as compared to ground and energised ends. Visual observation during the tests revealed that the arc propagates in both direction in the case of mid-region dry band which may result in low propagation velocity and high flashover voltage. These results shows that dry band loca-

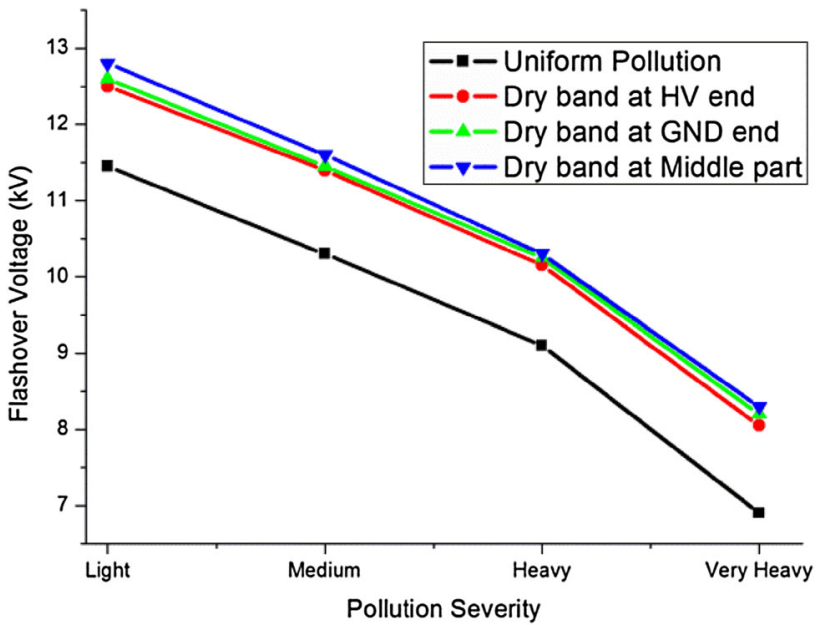

Fig. 5 Variation of flashover voltage with pollution severity and

tion effects the flashover characteristics of outdoor insulators. Figure 5 shows the relation between pollution severity, dry band location and flashover voltage. Propagation of flashover arc along the insulator surface was also effected by dry band location but was not investigated in this paper.

\section{Simulation results}

Numerical simulations are very important for understanding any physical process. A commercial simulation package, COMSOL Multiphysics was used to simulate the electric field and electric potential distribution along the four different configurations shown in Fig. 2. Finite element method (FEM) was implemented using the electrostatic physics of COMSOL Multiphysics. Power frequency AC voltage was applied to the copper hemisphere electrode, while copper plate was used as ground terminal. The sample was enclosed in a rectangular air box having dimensions of $14 \times 6 \times$ $1.4 \mathrm{~cm}^{3}$. The outer edges of the air box were assigned zero external current so that any external effect on electric field and potential distribution can be eliminated. The sample dimensions are $10 \times 4 \times 0.6 \mathrm{~cm}^{3}$. Pollution was modeled as a $2-\mathrm{mm}$ uniform water layer. Conductivity of the pollution layer was varied according to Table 1 to simulate different pollution conditions. Relative permittivity of silicone rubber and water was assumed to be 4.3 and 80 , respectively. All simulations were based on the experimental values of flashover voltages. Equations (6.1)-(6.5) were used in COMSOL Multiphysics to simulate the electric field [27].

$$
\begin{aligned}
& E=-\nabla V \\
& \nabla E=\frac{\rho}{\varepsilon} \\
& -\nabla(\nabla E)=\frac{\rho}{\varepsilon}
\end{aligned}
$$




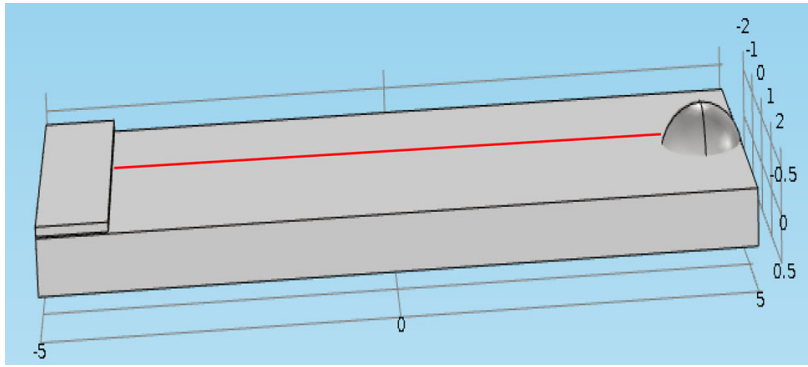

Fig. 6 Test sample used for simulation (all dimensions are in $\mathrm{cm}$ )

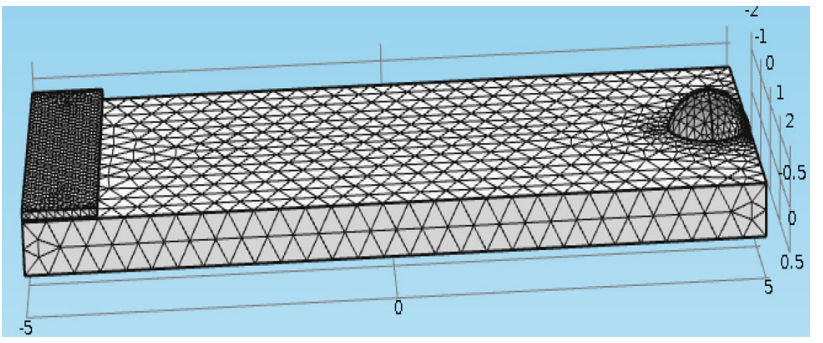

Fig. 7 Meshed sample (all dimensions are in $\mathrm{cm}$ )

$\varepsilon \nabla(\nabla V)=0$

$J=\sigma E$

In these equations, $\rho$ is the resistivity, $\varepsilon$ is the dielectric constant, $J$ is the current density and $\sigma$ is the conductivity of the pollution layer. Figures 6 and 7 show the sample configuration and meshing used for simulation. All the results obtained for electric field and potential were calculated along the cutline (red) as shown in Fig. 6.

\subsection{Electric field distribution}

Electric field calculation along an outdoor insulator is considered to be one of the most reliable diagnostic tools. Experimental and numerical investigations have been carried out previously to locate defects in outdoor insulators using electric field calculations [28,29]. In this paper, various contamination and dry band conditions were considered to calculate the electric field distribution along the surface of the silicone rubber insulator. All simulations are based on the experimental values of flashover voltage as shown in Tables 2 and 4. The results in Fig. 8 show the electric field distribution under various polluted and dry band conditions. Figure 8a shows the electric field distribution for a medium polluted sample. It can be seen from these results that electric field distribution is symmetrical and the highest electric field stress is found near the copper electrodes. Figure $8 \mathrm{~b}$ shows the electric field distribution when a dry band is inserted near the ground electrode. A dry band on the surface of an insulator acts as an open circuit and resists the flow of leakage current. The entire voltage drop occurs at the edge of the dry band so that electric field stress increases. This phenomenon is evident from the results of Fig. 8b. The electric field stress near the energised end is very low and is not enough to initiate an electrical discharge. In this case, the discharge will be initiated from the ground end as opposed to the energised end in the case of a uniform pollution layer. Figure $8 \mathrm{c}$ shows the electric field distribution along the surface of silicone rubber sample when a dry band is inserted near the energised electrode. The electric field distribution behaviour is quite similar to that of Fig. 8b. This time, however, the highest electric field stress is near the high-voltage electrode, and an electrical discharge (partial arc) will be initiated from the energised end. A similar behaviour was observed in Fig. 8d when a dry band was inserted at the middle of the silicone rubber sample. The highest electric field occurs at the middle part at the edge of the dry band. The electrical discharge will be initiated from the middle of the insulator and propagate in both directions. From experimental results, it was observed that the breakdown strength was high in this case which might be due to the propagation of discharge in both directions. The results obtained from numerical simulation are in close agreement with experimental results and the previous literature.

\subsection{Potential distribution}

Experimental results show that flashover voltage is greatly influenced by pollution severity and dry band location. To replicate this phenomenon, numerical simulations were carried out to calculate potential distribution along the insulator surface under different dry band conditions. It was previously investigated in [27] that potential distribution along the insulator surface is linear and is not much effected by pollution severity. Although the magnitude of flashover voltage is greatly affected by pollution severity, the distribution remains the same. For clean insulators, a very small amount of current (displacement current) flows through the insulator surface and the potential distribution is only dependent on self and stray capacitances. In the case of wet pollution, the magnitude of leakage current is high (conduction current) and changes with change in pollution severity that results in change in potential distribution. However, if the pollution severity is increased beyond a certain level, the potential distribution is unaffected by pollution severity. Figure 9 shows the potential distribution along the silicone rubber sample for a uniform pollution layer and dry bands at ground end, middle part and energised end. For uniform pollution layer, potential distribution was found to be linear. There is a sudden decrease in the potential distribution near the dry band and the distribution is non-linear as compared to that with a uniform pollution layer. These results show that dry band location has a direct influence on potential distribution along the surface of the insulator. These results are in close agree- 


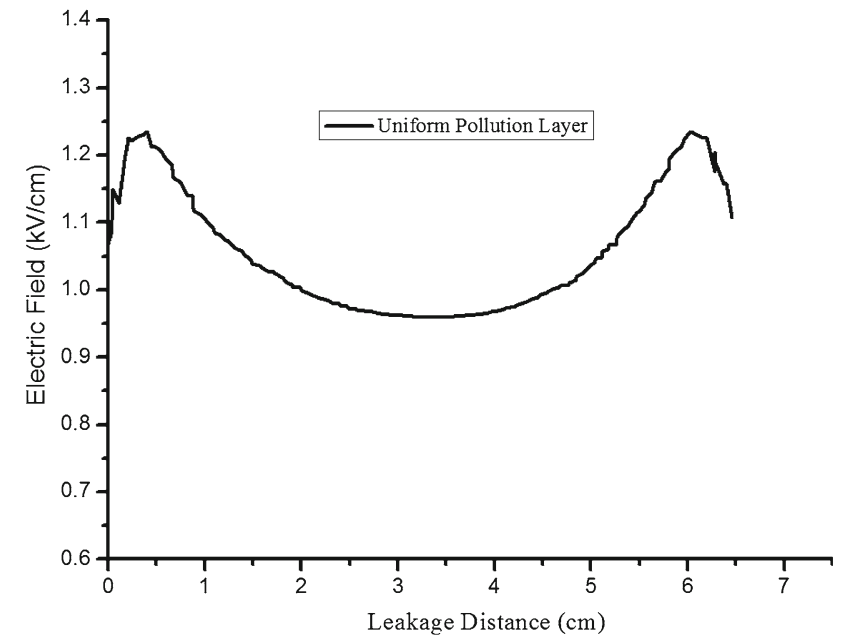

(a)

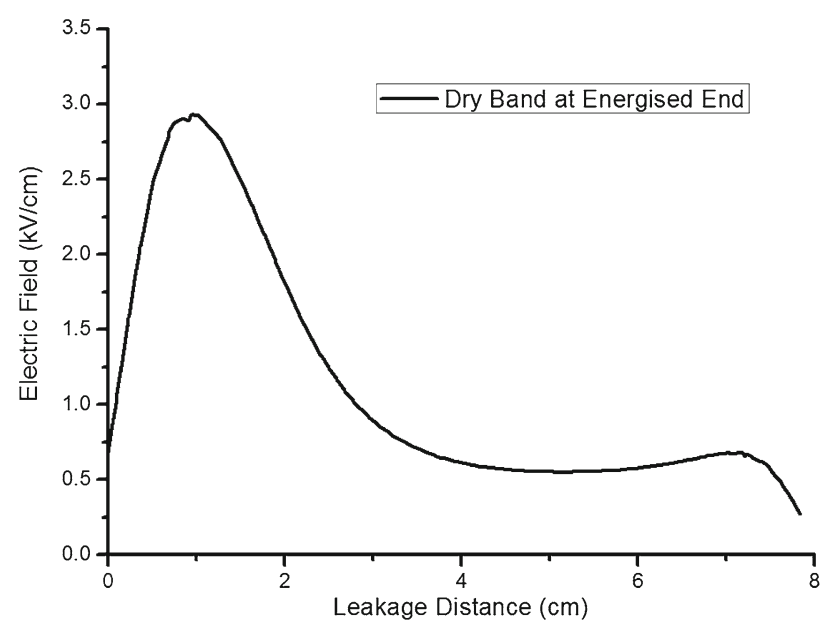

(c)

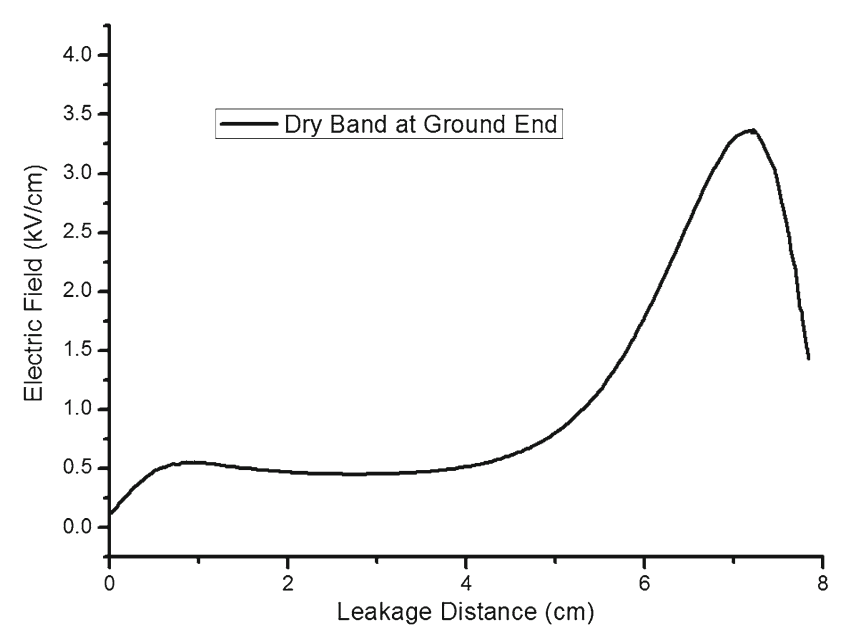

(b)

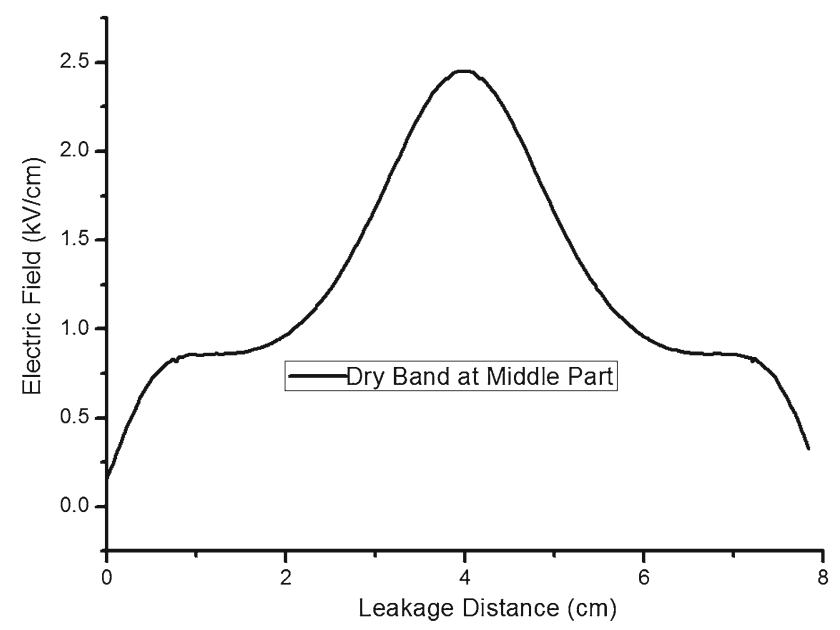

(d)

Fig. 8 Electric field distribution a uniform pollution layer, $\mathbf{b}$ dry band at ground end, $\mathbf{c}$ dry band at energised end, $\mathbf{d}$ dry band at middle part

ment with the experimental results and can be used for better understanding of flashover process along polluted insulators.

\section{Hydrophobicity recovery of silicone rubber}

Silicone rubber materials are well known for the recovery of hydrophobicity. During flashover tests, surface hydrophobicity decreases due to the high-energy flashover arcs. The electrical activity along the insulator surface fragments the polymer chains, especially along the smaller chains like LMW. Figure 10 shows the basic structure of LMW molecule. These molecules can either evaporate or bond with other molecules. It has been investigated previously that electrical activity ages the surface very rapidly [30]. In this paper, each flashover test lasted for 30-40 min on average. The samples were under high electric field stress during the test time.

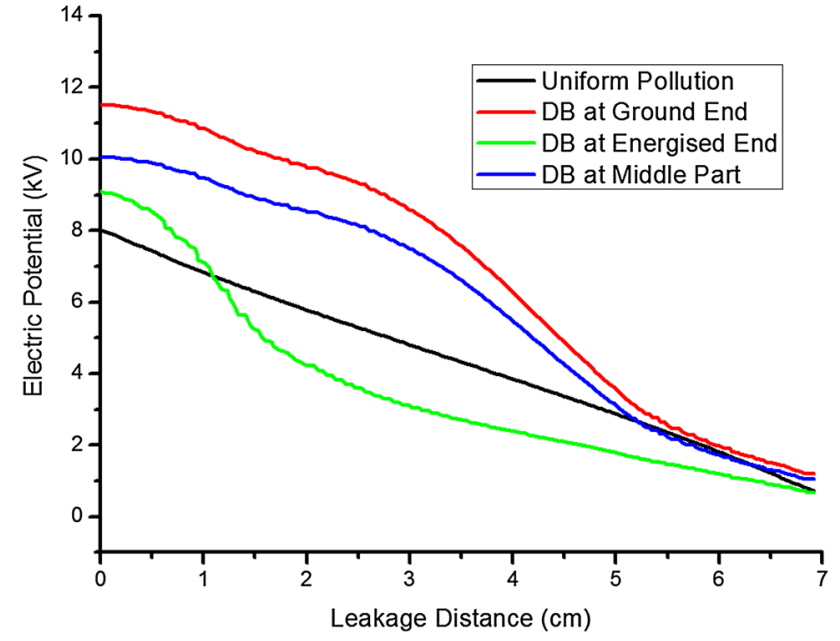

Fig. 9 Potential distribution a uniform pollution layer, $\mathbf{b}$ dry band at ground end, $\mathbf{c}$ dry band at energised end, $\mathbf{d}$ dry band at middle part 


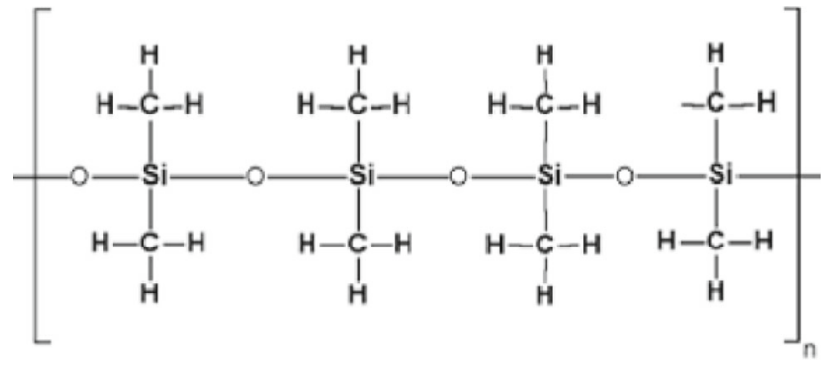

Fig. 10 Chemical structure of poly dimethyl siloxane, the LMW silicone rubber

Leakage current also flowed through the insulator surface and caused surface heating and dry band formation.

FTIR spectroscopy was used to study the surface degradation of silicone rubber samples after flashover tests and to investigate surface hydrophobicity. In FTIR spectroscopy, an infrared radiation is passed through a solid sample resulting in absorption as well as transmission of infrared radiations. The resulting spectrum from the absorption and transmission of infrared radiation creates a molecular fingerprint of the sample composition. In FTIR spectroscopy, no two unique molecules can create the same infrared spectrum [31]. An FTIR spectrometer (JASCO, FTIR-6100) was used to analyze silicone rubber samples after performing the flashover tests. The spectrometer resolution was set to $5 \mathrm{~cm}^{-1}$ and the wave number was in the range of $4000-500 \mathrm{~cm}^{-1}$. Initially, a virgin sample of silicone rubber was used to obtain the absorption spectrum and identify the important bands. Samples of $1 \times 1 \times 0.3 \mathrm{~cm}^{3}$ were cut from the ground end, energised end and middle part of the insulator to compare the ageing and degradation at different parts of the insulator surface. To study the hydrophobicity recovery property, readings were taken at four different times: immediately after flashover tests, and after 24, 48 and $72 \mathrm{~h}$.

The height of peak in an absorption spectrum indicates the concentration of chemical bonding [32,33]. A comparison of peak intensities was carried out between a virgin sample and the aged samples at specific time intervals. Several readings were taken at each time interval to improve the measurement accuracy. Readings were taken for a sample having uniform pollution layer with medium severity. During partial discharges, corona and flashover, the highenergy particles generated by the flashover arc can break the $\mathrm{Si}-\mathrm{O}$ bond in the material [34]. The advantage of this process is that breaking the $\mathrm{Si}-\mathrm{O}$ bond results in a large number of LMW molecules and this will help in the process of hydrophobicity recovery. These LMW molecules may migrate to the surface to restore hydrophobicity. Tables 5, 6 and 7 show the important bands and the corresponding peak heights for the virgin and polluted samples at the energised end, ground end and middle part, respectively. FTIR
Table 5 Absorbance peaks at energised end

Table 6 Absorbance peaks at ground end

Table 7 Absorbance peaks at middle part

\begin{tabular}{lllllll}
\hline Wavenumber $\left(\mathrm{cm}^{-1}\right)$ & Chemical bond & Virgin & $72 \mathrm{~h}$ & $48 \mathrm{~h}$ & $24 \mathrm{~h}$ & After the test \\
\hline $790-840$ & $\mathrm{Si}\left(\mathrm{CH}_{3}\right)_{2}$ & 0.98 & 0.85 & 0.81 & 0.77 & 0.72 \\
$1000-1100$ & $\mathrm{Si}-\mathrm{O}-\mathrm{Si}$ & 1.1 & 0.99 & 0.98 & 0.96 & 0.91 \\
$1255-1270$ & $\mathrm{Si}-\mathrm{CH}_{3}$ & 0.22 & 0.18 & 0.16 & 0.15 & 0.15 \\
$2960-2980$ & $\mathrm{CH}$ & 0.040 & 0.037 & 0.044 & 0.038 & 0.032 \\
$3500-3700$ & $\mathrm{OH}$ & 0.046 & 0.078 & 0.087 & 0.10 & 0.11 \\
\hline
\end{tabular}

\begin{tabular}{lllllll}
\hline Wavenumber $\left(\mathrm{cm}^{-1}\right)$ & Chemical bond & Virgin & $72 \mathrm{~h}$ & $48 \mathrm{~h}$ & $24 \mathrm{~h}$ & After the test \\
\hline $790-840$ & $\mathrm{Si}\left(\mathrm{CH}_{3}\right)_{2}$ & 0.98 & 0.88 & 0.82 & 0.8 & 0.74 \\
$1000-1100$ & $\mathrm{Si}-\mathrm{O}-\mathrm{Si}$ & 1.1 & 1.04 & 1.04 & 0.99 & 0.93 \\
$1255-1270$ & $\mathrm{Si}-\mathrm{CH}_{3}$ & 0.22 & 0.19 & 0.18 & 0.16 & 0.16 \\
$2960-2980$ & $\mathrm{CH}$ & 0.040 & 0.039 & 0.039 & 0.039 & 0.036 \\
$3500-3700$ & $\mathrm{OH}$ & 0.046 & 0.06 & 0.066 & 0.088 & 0.094 \\
\hline
\end{tabular}

\begin{tabular}{lllllll}
\hline Wavenumber $\left(\mathrm{cm}^{-1}\right)$ & Chemical bond & Virgin & $72 \mathrm{~h}$ & 48 & $24 \mathrm{~h}$ & After the test \\
\hline $790-840$ & $\mathrm{Si}\left(\mathrm{CH}_{3}\right)_{2}$ & 0.98 & 0.88 & 0.82 & 0.8 & 0.74 \\
$1000-1100$ & $\mathrm{Si}-\mathrm{O}-\mathrm{Si}$ & 1.1 & 1.05 & 1.06 & 0.99 & 0.93 \\
$1255-1270$ & $\mathrm{Si}-\mathrm{CH}_{3}$ & 0.22 & 0.19 & 0.18 & 0.16 & 0.17 \\
$2960-2980$ & $\mathrm{CH}$ & 0.040 & 0.039 & 0.036 & 0.035 & 0.036 \\
$3500-3700$ & $\mathrm{OH}$ & 0.046 & 0.062 & 0.066 & 0.088 & 0.094 \\
\hline
\end{tabular}


results from Tables 5, 6 and 7 show a decrease in the polymeric groups $\left(\mathrm{Si}-\mathrm{CH}_{3}, \mathrm{Si}\left(\mathrm{CH}_{3}\right)_{2}, \mathrm{Si}-\mathrm{O}-\mathrm{Si}\right.$ and $\left.\mathrm{CH}\right)$ and an increase in hydrophilic contents after the flashover tests. The hydrophilic group (OH radical) was observed to decrease with time because of hydrophobicity recovery, while the polymeric groups increase with time. Changes in the molecular content were more evident for the energised end, while a very small deviation was found for the ground end and middle part as compared to the virgin sample. Furthermore, it was observed that after $72 \mathrm{~h}$ of the flashover test, the hydrophobicity of the silicone rubber samples recovered up to some extent but not completely. The permanent loss of some of the polymeric molecules might be due to physical damage to the silicone rubber surface but that was not investigated in this paper.

\section{Discussions}

Flashover voltage and electric field distribution along outdoor insulators are influenced by many factors: two of them being pollution severity and dry band location. Pollution severity affects flashover voltage and electric field and it was observed that flashover occurs at lower electric field strength as pollution severity increases. Flashover voltage is also influenced by the rate of wetting, and the critical flashover voltage was much lower for a clean and wet insulator as compared to a clean and dry one. Leakage distance is one of the important parameters affecting the flashover voltage of outdoor insulators. Experimental results show a direct relationship between leakage distance and flashover voltage. Dry band formation along an outdoor insulator depends on various parameters and cannot be eliminated completely. Generally, dry bands form near the energised and ground electrodes because of the high electric field stress at these locations. Three different dry band configurations were considered in this paper and their effects on flashover voltage and electric field were investigated. Flashover voltage increases with the formation of dry bands and higher electric field stress is needed to initiate a discharge along the dry bands. In the case of a dry band in the middle part of the insulator surface, flashover voltage is higher than that of ground-end and energised-end dry bands. Numerical simulations of the proposed configuration show a similar behavior as that of the experimental results. Electric field distribution along a uniformly polluted insulator shows that high electric field stress occurs at the electrode end and that the overall distribution is symmetrical. When a dry band is inserted on the insulator surface, the distribution becomes asymmetrical and the highest electric field stress occurs at the edges of the dry bands instead of electrode end. The same behavior was observed in all three cases: dry band at energised end, ground end or middle part. Potential distribution along a uniformly polluted insulator shows the presence of a linear distribution irrespective of pollution severity. This may be because of the fact that a uniform pollution layer acts as a constant resistance layer which leads to linear potential distribution. In the case of dry band formation, potential distribution is nonlinear and a potential drop near the dry band was observed. Ageing of silicone rubber insulator in polluted environments is a very important phenomenon. Although flashover lasts for a few seconds, the high energy of flashover arc can break the molecular bonding of silicone molecules which can lead to loss of hydrophobicity. The hydrophobicity loss and recovery due to pollution flashover was investigated. Readings were taken at four different time intervals to allow enough time for relaxation and hydrophobicity recovery. FTIR readings taken immediately after flashover tests show a decrease in polymeric contents $\mathrm{Si}-\mathrm{CH}_{3}, \mathrm{Si}-\mathrm{O}-\mathrm{Si}, \mathrm{Si}\left(\mathrm{CH}_{3}\right)_{2}$ and an increase in hydrophilic groups corresponding to $\mathrm{O}-\mathrm{H}$ radicals as compared to the virgin sample. An increase in polymeric content and a decrease in hydrophilic content were observed in the FTIR results taken 24,48 and $72 \mathrm{~h}$ after the flashover tests. This shows the presence of hydrophobicity recovery for silicone rubber materials. This was more evident at the energised end as compared to the ground end and middle part. Even after $72 \mathrm{~h}$, complete recovery of hydrophobicity was not observed which shows that there is permanent damage along the silicone rubber surface. This may lead to ageing, degradation and reduction in the useful life of polymeric insulators.

\section{Conclusion}

Experimental investigation was carried out to assess the performance of silicone rubber material under contaminated and dry band conditions. Effects of pollution severity, leakage distance and dry band location on flashover voltage, electric field and ageing of silicone rubber samples were studied. It was shown experimentally that pollution severity reduces the breakdown strength of insulators and leads to flashover at lower electric field. This phenomenon was investigated numerically with COMSOL Multiphysics and was found in good agreement with the experimental results. Effect of leakage distance on flashover characteristics was also studied and a linear relation between leakage distance and flashover voltage was established. Dry bands were inserted at ground end, energised end and middle part of the insulator and flashover voltages were recorded. Dry band at the middle part has more influence on flashover voltage as compared to the other two locations. Numerical simulation of dry band locations shows that maximum electric field stress occurs at the edges of dry bands and not at the electrode ends as in the case of uniform pollution layer. Chemical changes on the surface of insulator due to high-energy partial arcs and flashover were 
investigated using FTIR spectroscopy. It was found that the polymeric contents in the silicone rubber surface decrease after flashover while the hydrophilic contents increase. FTIR data were taken at three different locations, ground end, energised end and middle part, and it was found that these changes were more evident at the energised end.

Hydrophobicity recovery property of silicone rubber was investigated by allowing rest time of 24,48 and $72 \mathrm{~h}$ after flashover tests and the results were in good agreement with the available literature. The output of this work will improve our knowledge regarding the flashover of contaminated insulators and increase the reliability of power transmission and distribution system specifically in polluted and humid environments. These results can also be used to improve the existing mathematical models for predicting the flashover voltage of polluted polymeric insulators.

Open Access This article is distributed under the terms of the Creative Commons Attribution 4.0 International License (http://creativecomm ons.org/licenses/by/4.0/), which permits unrestricted use, distribution, and reproduction in any medium, provided you give appropriate credit to the original author(s) and the source, provide a link to the Creative Commons license, and indicate if changes were made.

\section{References}

1. Amin M, Akbar M, Amin S (2007) Hydrophobicity of silicone rubber used for outdoor insulation (an overview). Rev Adv Mater Sci 16:10-26

2. Youn BH, Hu CS (2005) Surface degradation of HTV silicone rubber and EPDM used for outdoor insulators under accelerated ultra violet weathering condition. IEEE Trans Dielect Electr Insul 12:1015-1023

3. Du B, Li Z (2015) Hydrophobicity, surface charge and DC flashover characteristics of direct-fluorinated RTV silicone rubber. IEEE Trans Dielect Electr Insul 22(2):934-940

4. Haddad G, Wong KL, Gupta RK (2014) Dielectric breakdown characteristics of HTV silicone rubber under multiple stress conditions. In: IEEE international conference on electrical insulating materials (ISEIM)

5. Vlastos AE, Sheriff EM (1989) Experience from insulators with silicone rubber sheds and shed coatings. In: IEEE transactions on power delivery, Paper 89, WM 121-5 PWRD

6. Haddad G, Gupta RK, Wong KL (2012) Study of surface degradation of HTV silicone rubber under thermal and ultraviolet exposures. In: IEEE international conference on high voltage engineering and application (ICHVE)

7. Hillborg H, Gedde UW (2012) Oxidative surface treatment of silicone rubber. Silicone surface science. Springer, Amsterdam, pp 299-318

8. Farhadinejad Z, Ehsani M, Ahmadi-Joneidi L, Shayegani AA, Mohseni H (2012) Effects of UVC radiation on thermal, electrical and morphological behavior of silicone rubber insulators. IEEE Trans Dielectr Electr Insul 19(5):1740-1749

9. Zhu Y, Ma B, Liu X, Song B, Zhang X (2013) Aging performance of silicone rubber exposed to UV and sandstorm. In: IEEE conference on electrical insulation and dielectric phenomena (CEIDP)

10. Hackam R (1999) Outdoor HV composite polymeric insulators. IEEE Trans Dielectr Electr Insul 6:557-585
11. Karady GG (1999) Flashover mechanism of non-ceramic insulators. IEEE Trans Dielectr Electr Insul 6(5):718-723

12. Shah M, Karady GG, Brown RL (1995) Flashover mechanism of silicone rubber insulators used for outdoor insulation-II. IEEE Trans Power Deliv 10(4):1972-1978

13. Moreno VM, Gorur RS (2001) Effect of long-term corona on nonceramic outdoor insulator housing materials. In: IEEE transactions on dielectrics and electrical insulation, vol 8.1, pp 117-128

14. Boheme H, Obenaus F (1966) Pollution flashover tests on insulators in the laboratory and in systems and the model concept of creepage path flashover, Cigre, p 407

15. Wilkins R (1969) Flashover voltage of high-voltage insulators with uniform surface-pollution films. Proc Inst Electr Eng 116(3):457465

16. Sundararajan R, Gorur RS (1993) Dynamic arc modeling of pollution flashover of insulators under dc voltage. IEEE Trans Electr Insul 28(2):209-218

17. Topalis FV, Gonos IF, Stathopulos IA (2001) Dielectric behavior of polluted porcelain insulators. IEE Proc Gener Transm Distrib 148(4):269-274

18. Ghosh PS, Chatterjee N (1995) Polluted insulator flashover model for ac voltage. IEEE Trans Dielectr Electr Insul 2(1):128-136

19. IEC 60507 (1993) Artificial pollution tests on high-voltage insulators to be used on A.C. systems

20. Dong B, Jiang X, Hu J, Shu L, Sun C (2012) Effects of artificial polluting methods on $\mathrm{AC}$ flashover voltage of composite insulators. IEEE Trans Dielectr Electr Insul 19(2):714-722

21. Gutman I, Dernfalk A (2010) Pollution tests for polymeric insulators made of hydrophobicity transfer materials. IEEE Trans Dielectr Electr Insul 17(2):384-393

22. Williams DL, Haddad A, Rowlands AR, Young HM, Waters RT (1999) Formation and characterization of dry bands in clean fog on polluted insulators. IEEE Trans Dielectr Electr Insul 6(5):724-731

23. Chrzan KL, Moro F (2007) Concentrated discharges and dry bands on polluted outdoor insulators. IEEE Trans Power Deliv 22(1):466471

24. Jian-bo Z, Bo G, Qiao-gen Z (2010) Dry band formation and its influence on electric field distribution along polluted insulator. In:IEEE Asia-Pacific power and energy engineering conference (APPEEC), pp 1-5

25. Kim SH, Cherney E, Hackam R, Rutherford KG (1994) Chemicalchanges at the surface of RTV silicone rubber coatings on insulatorsduring dry-band arcing. IEEE Trans Dielectr Electr Insul 1(1):106-123

26. Waters RT, Haddad A, Griffiths H, Harid N, Sarkar P (2010) Partialarc and spark models of the flashover of lightly polluted insulators. IEEE Trans Dielectr Electr Insul 17(2):417-424

27. Doshi T, Gorur RS, Hunt J (2011) Electric field computation of composite line insulators up to $1200 \mathrm{kV}$ AC. IEEE Trans Dielectr Electr Insul 18(3):861-867

28. Tzimas A, Zachariades C, Rowland SM (2013) Electric field analysis of $132 \mathrm{kV}$ EPDM insulator and correlation with ageing features. In: IEEE electrical insulation conference (EIC), vol 2013, pp 210, 214, 2-5 June 2013

29. Arshad, Nekahi A, McMeekin SG, Farzaneh M (2015) Effect of pollution severity on electric field distribution along a polymeric insulator. In: 11th international conference on the properties and applications of dielectric materials (ICPADM)

30. Kim SH, Cherney EA, Hackman R (1992) Hydrophobic behaviour of insulators coated with RTV silicone rubber. In: IEEE transactions an electrical insulation, vol 27, 3 June 1992, pp 610-622

31. Griffiths PR, De Haseth JA (2007) Fourier transform infrared spectrometry, vol 171. Wiley, New York

32. Sörqvist T, Vlastós AE (1997) Performance and ageing of polymeric insulators. IEEE Trans Power Deliv 12(4):1657-1665 
33. Sundararajan R, Mohammed A, Chaipanit N, Karcher T, Liu Z (2004) In-service aging and degradation of $345 \mathrm{kV}$ EPDM transmission line insulators in a coastal environment. IEEE Trans Dielectr Electr Insul 11(2):348-361
34. Liu H, Cash G, Sovar RD, George G, Birtwhistle D (2006) Studies of the diffusion of low molecular weight silicone fluids on polluted hv silicone insulators. I. Use of diffuse reflectance FTIR. IEEE Trans Dielectr Electr Insul 13(4):877-884 